\title{
Capacidade produtiva local de Tectona grandis em Monte Dourado, Estado do Pará, Brasil
}

\author{
Local productive capacity of Tectona grandis in Monte Dourado, Pará State, Brazil
}

\author{
Fábio Xavier da Conceição ${ }^{\mathrm{I}}$ Ronaldo Drescher ${ }^{\mathrm{II}}$ Allan Libanio Pelissari' ${ }^{\mathrm{II}}$ \\ Luciano Rodrigo LanssanovaII Cyro Matheus Cometti Favalessa ${ }^{\text {II }}$ José Guilherme Roquette ${ }^{\text {II }}$
}

\section{RESUMO}

O trabalho teve como objetivo determinar a capacidade produtiva local da teca em Monte Dourado, Estado do Pará, Brasil, por meio da análise de curvas de índice de sítio. Os dados foram obtidos pela análise de tronco de dez árvores dominantes de um povoamento com 26 anos de idade. Foram ajustados dez modelos de regressão e o critério de seleção obedeceu ao menor erro padrão da estimativa, ao maior coeficiente de determinação ajustado, à significância do teste $F$ e dos coeficientes de regressão e à análise gráfica dos resíduos. As curvas de índice de sítio foram confeccionadas pelo método da curva-guia. O modelo de Spillman apresenta o melhor ajuste, por meio do qual foram geradas três curvas de índice de sítio de 16m a 24m na idade-índice de 26 anos. A região de Monte Dourado, PA, apresenta potencial produtivo para o desenvolvimento da teca.

Palavras-chave: teca, índice de sítio, altura dominante.

\section{ABSTRACT}

The study aims to determine the local productive capacity of teak in Monte Dourado, Pará State, Brazil, with the analysis of site index curves. The data were obtained by stem analysis of ten dominant trees in a stand with 26 years of age. Were adjusted ten regression models and the criterion for selection followed the lowest standard error of estimate, the highest adjusted coefficient of determination, the significance of $F$ test and of regression coefficients and graphical analysis of the residuals. The site index curves were built by the guide-curve method. The Spillman model present the best fit, by which were generated three curves of site index from $16 \mathrm{~m}$ to $24 \mathrm{~m}$, at 26 years of index-age. The region of Monte Dourado - PA showed a productive potential for the development of teak.

Key words: teak, site index, dominant height.

\section{INTRODUÇÃO}

A teca (Tectona grandis L.f.) é uma espécie nativa do continente asiático, sendo introduzida em diversas regiões da África e Américas, ocupando espaço de destaque entre as principais espécies produtoras de madeira tropical para o mercado de móveis de luxo e construção naval (PANDEY \& BROWN, 2000; BERMEJO et al., 2004; FIGUEIREDO et al., 2005).

A espécie apresenta maior taxa de crescimento em localidades com precipitação anual de $1.250 \mathrm{~mm}$ a $3.750 \mathrm{~mm}$, associada a um período de três a cinco meses de seca e temperatura mínima de $13^{\circ} \mathrm{C}$ a $17^{\circ} \mathrm{C}$ e máxima de $39^{\circ} \mathrm{C}$ a $43^{\circ} \mathrm{C}$. Adapta-se a uma grande variedade de solos, porém tem preferência pelos de textura franco-arenosos a argilosos, profundos, bem drenados e férteis

\footnotetext{
IFaculdade de Engenharia Florestal (FENF), Universidade Federal de Mato Grosso (UFMT), Av. Fernando Correa da Costa, 2367, Boa Esperança, 78060-900, Cuiabá, MT, Brasil. E-mail: fabioxavier@florestal.eng.br. Autor para correspondência.

IPrograma de Pós-graduação em Ciências Florestais e Ambientais (PPGCFA), FENF, UFMT, Cuiabá, MT, Brasil.

IIIPrograma de Pós-graduação em Engenharia Florestal (PPGEF), Universidade Federal do Paraná (UFPR), Curitiba, PR, Brasil.
} 
(KAOSA-ARD, 1998; PANDEY \& BROWN, 2000; MONTERO et al., 2001).

As estimativas da produtividade de um determinado local são elementos fundamentais para o manejo florestal, pois contribuem para a determinação da colheita anual, do período de rotação e da escolha de espécies adequadas (VARGAS-LARRETA et al., 2010). Há vários métodos descritos para proceder à classificação da capacidade produtiva de sítios florestais, entretanto, a altura dominante correlacionada com a idade tem sido considerado o método mais prático e usual (SELLE et al., 2008), uma vez que esta variável representa a resposta aos fatores ambientais inter-relacionados e não sofre significativa influência dos tratamentos silviculturais e da competição (TONINI et al., 2002).

$\mathrm{O}$ interesse na teca como uma alternativa aos plantios florestais tradicionais no Brasil vem crescendo muito atualmente (SCHUHLI \& PALUDZYSZYN FILHO, 2010). No entanto, apesar de diversos estudos sobre a espécie no mundo, estes ainda são escassos no Brasil (TONINI et al., 2009), principalmente de avaliações em períodos próximos ao ciclo de produção da espécie. Assim, o objetivo do presente trabalho foi determinar a capacidade produtiva local da teca em Monte Dourado, Estado do Pará, por meio da análise de curvas de índice de sítio.

\section{MATERIAL E MÉTODOS}

O estudo foi realizado em uma propriedade da empresa Jari Celulose, situado no município de Monte Dourado, Estado do Pará, a $453 \mathrm{~km}$ da capital Belém, em um povoamento equiâneo de teca com 26 anos de idade, consorciado com Gmelina aborea Roxb., intercalando uma linha de teca com uma de gmelina, no espaçamento de $6 \mathrm{~m} \times 2 \mathrm{~m}$. O clima da região é caracterizado como Equatorial Quente Úmido, com uma estação chuvosa de janeiro a julho e outra seca de agosto a dezembro. A temperatura média mensal é de $26^{\circ} \mathrm{C}$, com máxima de $34^{\circ} \mathrm{C}$ e mínima de $22^{\circ} \mathrm{C}$. Avegetação predominante é a Floresta Ombrófila Densa e o solo é caracterizado como Latossolo Amarelo (JARI CELULOSE, 2009).

Para a coleta de dados e posterior análise de tronco, foram abatidas dez árvores dominantes com os discos coletados em posições fixas de $0,10 \mathrm{~m} ; 1,30 \mathrm{~m}$ e em distâncias de dois metros ao longo do fuste até a altura total, totalizando 260 pares de dados (altura dominante $\mathrm{x}$ idade).

Foram ajustados dez modelos de regressão para a estimativa da altura dominante em função da idade (Tabela 1), disponíveis na literatura florestal (SELLE et al., 2008; HESS \& SCHNEIDER, 2009; MILANI, 2010). O critério de seleção obedeceu, inicialmente, ao menor erro padrão da estimativa em porcentagem $\left(\mathrm{S}_{\mathrm{yx}} \%\right)$ e ao maior coeficiente de determinação ajustado $\left(\mathrm{R}_{\text {aj. }}^{2}\right)$. Também foi avaliada a significância do teste $\mathrm{F}$ e dos coeficientes de regressão (â), em nivel de 5\% de probabilidade, e a análise gráfica dos resíduos.

No caso dos modelos logarítmicos (1 a 7, Tabela 1), a discrepância, ao se efetuar a operação inversa para se obter a variável de interesse, foi corrigida multiplicando-se o valor estimado pelo fator de correção de Meyer (MACHADO et al., 2008), expresso por: $F C M=e^{0,5 \cdot\left(S_{y x}\right)^{2}}$, em que: $F C M=$ fator de correção de Meyer; $\mathrm{e}=$ exponencial; $\mathrm{e}_{\mathrm{yx}}=$ erro padrão de estimativa.

As classes de sítio foram determinadas por meio da metodologia proposta por SCHNEIDER (1993), com o método da curva guia e a idade-índice de 26 anos.

\section{RESULTADOS E DISCUSSÃO}

Os ajustes proporcionaram medidas de erro padrão de estimativa em porcentagem $\left(\mathrm{S}_{\mathrm{yx}} \%\right)$ superiores a $11 \%$ (Tabela 2), sendo que os modelos Backman modificado (3) e Prodan (10) forneceram os maiores valores, de $16,705 \%$ e $14,267 \%$, enquanto os modelos Hoerl (4) e Chapman-Richards (8) apresentaram os menores $\mathrm{S}_{\mathrm{yx}} \%$, com $11,653 \%$ e $11,539 \%$, respectivamente.

Todos os modelos apresentaram coeficientes de determinação ajustado $\left(\mathrm{R}_{\mathrm{aj}}{ }_{\mathrm{aj}}\right)$ superiores a 0,5 (Tabela 2), com o menor valor de 0,575 para o modelo Backman modificado (3) e o maior para o Spillman (7), com 0,797, enquanto os valores da estatística $F$ e dos coeficientes de regressão (ß) foram todos significativos (Tabela 2). No entanto, os modelos de Backman (2), Hoerl (4), Spillman (7) e Chapman- 
Tabela 1 - Modelos de altura dominante ajustados para Tectona grandis em Monte Dourado, PA.

\begin{tabular}{lll}
\hline $\mathrm{N}^{\mathrm{o}}$ & \multicolumn{1}{c}{ Modelo } & Denominação \\
\hline 1 & $\ln (H d)=\beta_{0}+\beta_{1}\left(\frac{1}{t}\right)+\varepsilon_{i}$ & Shumacher \\
2 & $\ln (H d)=\beta_{0}+\beta_{1} \cdot \ln (t)+\beta_{2} \cdot \ln (t)^{2}+\varepsilon_{i}$ & Backman \\
3 & $\ln (H d)=\beta_{1} \cdot \ln (t)+\beta_{2} \cdot \ln (t)^{2}+\varepsilon_{i}$ & Backman modificado \\
4 & $\ln (H d)=\beta_{0}+\beta_{1} \cdot\left(\frac{1}{t}\right)+\beta_{2} \cdot \ln (t)+\varepsilon_{i}$ & Hoerl \\
5 & $\ln (H d)=\beta_{0}+\beta_{1}(t)+\beta_{2}(t)^{2}+\beta_{3}(t)^{3}+\varepsilon_{i}$ & Moissev \\
6 & $\ln (H d)=\beta_{0}+\beta_{1} \cdot \ln (t)+\beta_{2}(t)+\varepsilon_{i}$ & Gram \\
7 & $\ln (H d)=\beta_{0}+\beta_{1}\left(\frac{1}{t}\right)+\beta_{2}\left(\frac{1}{t}\right)^{2}+\beta_{3}\left(\frac{1}{t}\right)^{3}+\varepsilon_{i}$ & Spillman \\
8 & $H d=\beta_{0}\left(1-e^{\left(-\beta_{1} \cdot t\right)}\right)_{2}^{\beta_{2}}+\varepsilon_{i}$ & Chapman-Richards \\
9 & $H d=\beta_{0}\left(1-e^{\left(-\beta_{1} \cdot t\right)}\right)+\varepsilon_{i}$ & Mitscherlich \\
10 & $H d=\frac{t^{2}}{\beta_{0}+\beta_{1} \cdot t+\beta_{2} \cdot t^{2}}+\varepsilon_{i}$ & Prodan
\end{tabular}

$\mathrm{Hd}=$ altura dominante $(\mathrm{m}) ; \mathrm{t}=$ idade $(\operatorname{anos}) ; \beta=$ coeficiente de regressão; $\ln =$ logaritmo neperiano; e $\varepsilon_{\mathrm{i}}=$ erro associado.

Richards (8) forneceram os melhores ajustes e, para estes, foram confeccionados os gráficos de distribuição dos resíduos em função da altura dominante estimada (Figura 1).

A análise gráfica dos resíduos demonstrou que a amplitude e a distribuição residual dos modelos foram semelhantes, com tendência a superestimar as menores alturas entre o intervalo de $5 \mathrm{~m}$ a $10 \mathrm{~m}$ nos modelos de Backman (Figura 1A), Hoel (Figura 1B) e Chapman-Richards (Figura 1D). Já o modelo de Spillman (Figura 1C) forneceu a melhor distribuição residual com ausência de tendenciosidade.

Assim, a partir da equação $\ln (\mathrm{Hd})=3,2595$ - 7,0972.(1/t) + 20,7287.(1/t) $)^{2}-24,7256 .(1 / t)^{3}$, os coeficientes $\hat{a}_{1}$, $\hat{a}_{2}$ e $\hat{a}_{3}$ foram fixados, gerados novos interceptos $\left(\hat{\mathrm{a}}_{0}\right)$ e confeccionadas três curvas nas

Tabela 2 - Coeficientes de regressão $\left(\beta_{0}, \beta_{1}, \beta_{2}\right.$ e $\left.\beta_{3}\right)$, erro padrão de estimativa em porcentagem $\left(S_{y x} \%\right)$, coeficiente de determinação ajustado $\left(\mathrm{R}_{\text {aj. }}^{2}\right)$ e teste $\mathrm{F}$ dos modelos de altura dominante ajustados para Tectona grandis em Monte Dourado, PA.

\begin{tabular}{lccccccc}
\hline Modelo & $\beta_{0}$ & $\beta_{1}$ & $\beta_{2}$ & $\beta_{3}$ & $\mathrm{~S}_{\mathrm{yx}} \%$ & $\mathrm{R}_{\text {aj. }}^{2}$ & $\mathrm{~F}$ \\
\hline 1 & $3,0498^{*}$ & $-2,5886^{*}$ & & & 12,670 & 0,756 & $962,02^{*}$ \\
2 & $1,3847^{*}$ & $0,8231^{*}$ & $-0,1000^{*}$ & & 11,731 & 0,791 & $538,78^{*}$ \\
3 & & $2,1252^{*}$ & $-0,3812^{*}$ & & 16,705 & 0,575 & $19770,50^{*}$ \\
4 & $2,4708^{*}$ & $-1,4720^{*}$ & $0,1839^{*}$ & & 11,653 & 0,793 & $563,91^{*}$ \\
5 & $1,6949^{*}$ & $0,1844^{*}$ & $-0,0095^{*}$ & $0,0002^{*}$ & 12,224 & 0,773 & $295,35^{*}$ \\
6 & $1,5787^{*}$ & $0,5900^{*}$ & $-0,0193^{*}$ & & 11,794 & 0,788 & $515,43^{*}$ \\
7 & $3,2595^{*}$ & $-7,0972^{*}$ & $20,7287^{*}$ & $-24,7256^{*}$ & 11,539 & 0,797 & $392,44^{*}$ \\
8 & $22,7403^{*}$ & $0,0669^{*}$ & $0,5334^{*}$ & & 11,702 & 0,792 & $5659,24^{*}$ \\
9 & $20,0251^{*}$ & $0,1647^{*}$ & & & 12,422 & 0,765 & $7520,16^{*}$ \\
10 & $-0,4030^{*}$ & $0,2631^{*}$ & $-0,0044^{*}$ & & 14,267 & 0,690 & $3783,16^{*}$ \\
\hline
\end{tabular}

\footnotetext{
* = significância a $5 \%$
} 


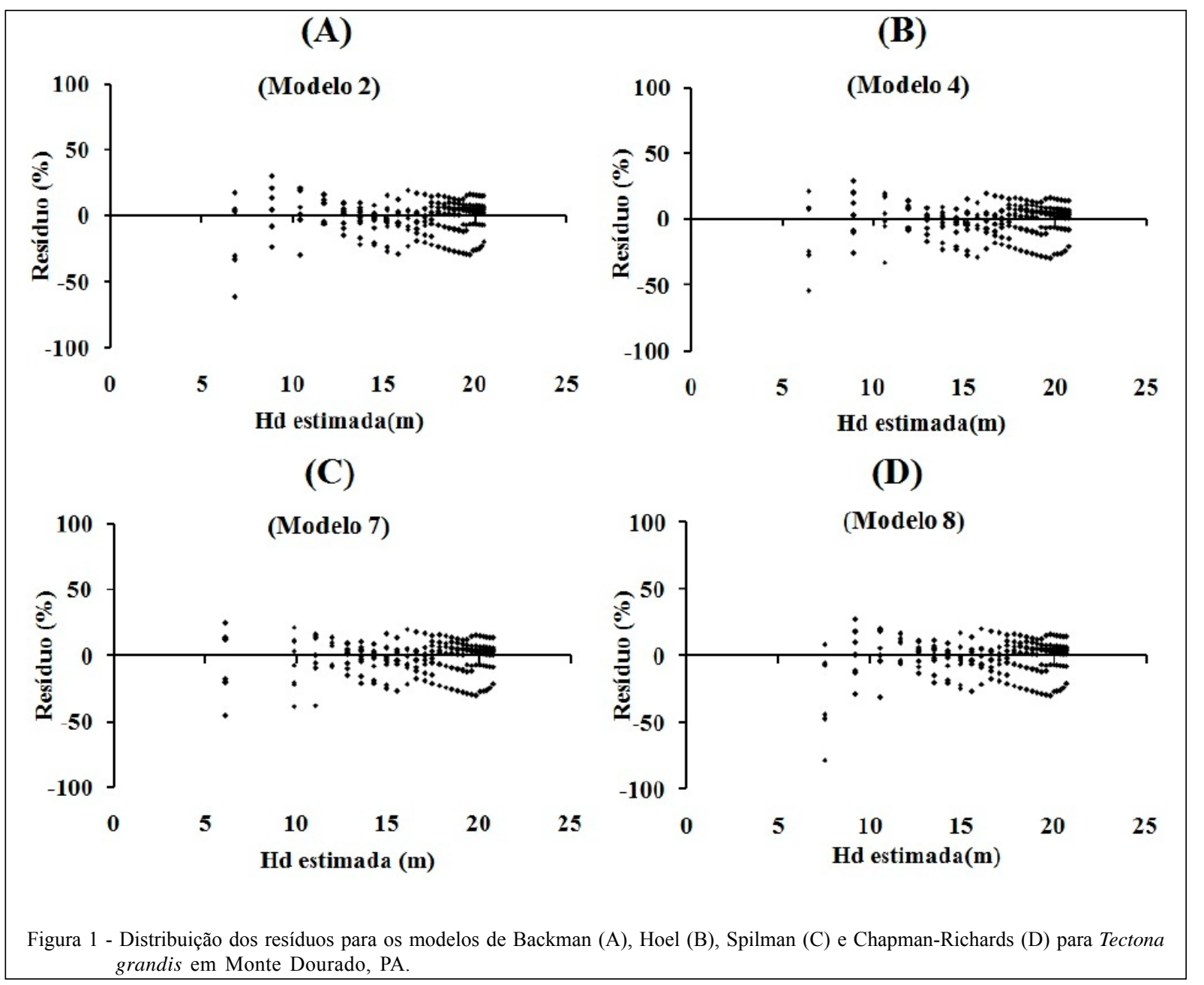

classes de sítio de $16 \mathrm{~m}, 20 \mathrm{~m}$ e $24 \mathrm{~m}$ na idade-índice de 26 anos (Figura 2), as quais descreveram com boa precisão as trajetórias de crescimento em altura dominante.

O crescimento inicial foi inferior ao observado por CRUZ et al. (2008) em Tangará da Serra, Mato Grosso, em um povoamento de teca com 4 anos e com alturas dominantes de $8 \mathrm{~m}$ e $15 \mathrm{~m}$, para o pior e o melhor sítio, respectivamente. No entanto, foram semelhantes aos observados por DRESCHER (2004) em Brasnorte e Santo Antônio do Leverger, Mato Grosso, com alturas dominantes de 20m, 18m, 16m, 14m, $12 \mathrm{~m}$ e $10 \mathrm{~m}$ para seis classes de sítio na idade-índice de 10 anos.

De maneira geral, os valores foram superiores aos obtidos por diversos autores em povoamentos de teca nos países asiáticos, como KADAMBI (1993) e
JHA (1999) na Índia, com alturas dominantes de 20m nos melhores sítios, $8 \mathrm{~m}$ nos médios e $2 \mathrm{~m}$ nos piores na idade de referência de 20 anos; e SAJJADUZZAMAN et al. (2005), em Bangladesh, com 20m e 22m em duas classes de sítio aos 40 anos. Isso evidencia a boa capacidade produtiva dos sítios da região de Monte Dourado - PA.

\section{CONCLUSÃO}

O modelo de Spillman e o método da curva guia são eficientes para determinar a capacidade produtiva local da teca (Tectona grandis) em Monte Dourado, PA, o qual apresenta adequado potencial produtivo para o estabelecimento de povoamentos da espécie. 


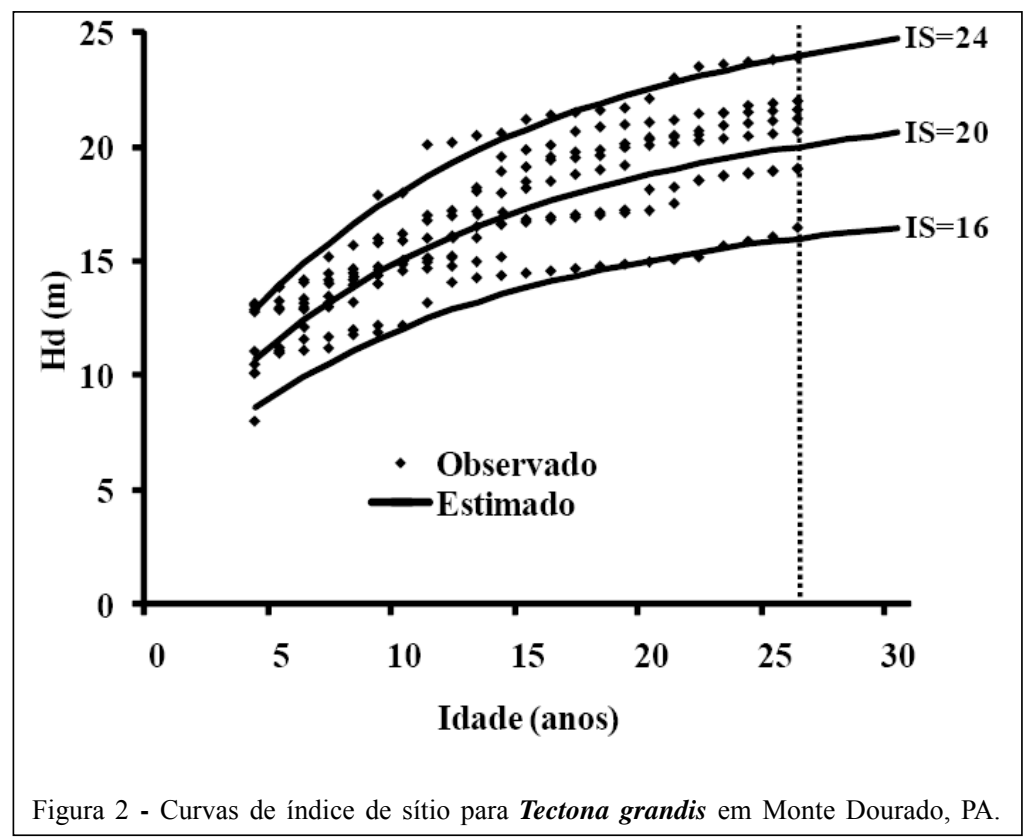

\section{REFERÊNCIAS}

BERMEJO, I. et al. Growth and yield models for teak plantations in Costa Rica. Forest Ecology and Management, v.189, p.97-110, 2004. Disponível em: <http://www2.montes.upm.es/ Dptos/DptoSilvopascicultura/SanMiguel/pdfs/publicaciones/ 2004_Tablas\%20prod\%20teca\%20Costa\%20Rica\%202004.pdf>. Acesso em: 4 jun. 2011. doi: 10.1016/j.foreco.2003.07.031.

CRUZ, J.P. da et al. Curvas de crescimento e de índice de local para povoamentos de Tectona grandis em Tangará da Serra, Mato Grosso. Revista Árvore, v.32, n.4, p,679-685, 2008. Disponível em: <http://www.scielo.br/pdf/rarv/v32n4/ a09v32n4.pdf>. Acesso em: 5 jun. 2011. doi: 10.1590/S010067622008000400009 .

DRESCHER, R. Crescimento e produção de Tectona grandis Linn. F., em povoamentos jovens de duas regiões do Estado de Mato Grosso. 2004. 133f. Tese (Doutorado em Engenharia Florestal) - Universidade Federal de Santa Maria, Santa Maria. Disponível em: <http://cascavel.cpd.ufsm.br/tede/ tde_arquivos/10/TDE-2006-12-01T142839Z-255/Publico/ RONALDODRESCHER.pdf>. Acesso em: 4 jun. 2011.

FIGUEIREDO, E.O. et al. Análise econômica de povoamentos não desbastados de Tectona grandis L.f., na microrregião do baixo Rio Acre. Cerne, v.11, n.4, p.342-353, 2005. Disponível em: <http://redalyc.uaemex.mx/pdf/744/ 74411404.pdf\%20>. Acesso em: 1 jun. 2011.

HESS, A.F.; SCHNEIDER, P.R. Crescimento em altura de Araucaria angustifolia (Bertol.) Kuntze em três locais do Rio Grande do Sul. Ambiência, v.5, n.2, p.213-232, 2009. Disponível em: <http://www.unicentro.br/editora/revistas/ ambiencia/v5n2/artigo\%203.pdf>. Acesso em: 2 jun. 2011.

JARI CELUlOSE. Avaliação do manejo florestal das plantações da Jari Celulose S.A. Monte Dourado, 2009. 22p. Disponível em: <http://www.scscertified.com/nrc/ certificates/forest_jari_port.pdf $>$. Acesso em: 5 jun. 2011.

JHA, K.K. Teak (Tectona grandis) farming. Uttar Pradesh: International Book Distributing, 1999. 125p.

KADAMBI, K. Silviculture and management of teak. Dehra Dun: Natraj Publishers, 1993. 137p.

KAOSA-ARD, A. Overview of problems in teak plantation establishment. In: REGIONAL SEMINAR ON TEAK, 2., 1998, Yangon. Proceeding... Bangkok: FAO, 1998. p.49-59. Disponível em: <http://www.fao.org/DOCREP/005/AC773E/ ac773e08.htm>. Acesso em: 5 jun. 2011.

MACHADO, S.A. et al. Comportamento da relação hipsométrica de Araucaria angustifolia no capão da Engenharia Florestal da UFPR. Pesquisa Florestal Brasileira, n.56, p.5-16, 2008. Disponível em: <http://www.cnpf.embrapa.br/ publica/pfb-revista-antiga/pfb_56/1_S_A_Machado(2).pdf $>$. Acesso em: 7 jun. 2011.

MILANI, E.J. Crescimento de Podocarpus lambertii Klotzsch ex Endl. em duas regiões fitogeográficas no Estado do Rio Grande do Sul. 2010. 155f. Tese (Doutorado em Engenharia Florestal) - Universidade Federal de Santa Maria, Santa Maria. Disponível em: <http://www.vsdani.com/ppgef/tesesdissertacoes/ 8e42eerni_jos_milani_tese_de_doutorado.pdf $>$. Acesso em: 6 jun. 2011.

MONTERO, M.M. et al. Relación del índice de sitio con los factores que influyen en el crescimento de Tectona grandis L. F. y Bombacopsis quinata (Jacq.) Dugand, en Costa Rica. Revista Forestal Centroamericana, n.35, p.13-18, 2001. Disponível em: <http://web.catie.ac.cr/informacion/RFCA/ rev35/pagina13-18.pdf>. Acesso em: 5 jun. 2011. doi: 10.1590/ S0100-06832006000500001. 
PANDEY, D.; BROWN, C. Teak: a global overview. Unasylva, v.51, n.201, p.3-11, 2000. Disponível em: <http://www.fao.org/ forestry/4602-06dd4a 3ffc3583aae26be6c4cc5ef851a.pdf $>$. Acesso em: 3 jun. 2011.

SAJJADUZZAMAN, M. et al. Site index for teak (Tectona grandis Linn F.) in forest plantations of Bangladesh. International Journal of Agriculture and Biology, v.7, n.4, p.547-549, 2005. Disponível em: <http:// www.fspublishers.org/ijab/past-issues/IJABVOL_7_NO_4/ 4.pdf>. Acesso em: 4 jun. 2011.

SCHNEIDER, P.R. Introdução ao manejo florestal. Santa Maria: UFSM/CEPEF/FATEC, 1993. 348p.

SCHUHLI, G.S.; PALUDZYSZYN FILHO, E. O cenário da silvicultura de teca e perspectivas para o melhoramento genético. Pesquisa Florestal Brasileira, v.30, n.63, p.217-230, 2010 Disponível em: <http://www.cnpf.embrapa.br/pfb/index.php/pfb/ article/view/84/148>. Acesso em: 5 jun. 2011. doi: 10.4336 2010.pfb.30.63.217.

SELLE, G.L. et al. Como classificar sítios florestais através da altura dominante do povoamento. Colombo: Embrapa
Florestas, 2008. 46p. Disponível em: <http:// www.cnpf.embrapa.br/publica/seriedoc/edicoes/Doc166.pdf>. Acesso em: 4 jun. 2011

TONINI, H. et al. Comparação gráfica entre curvas de índice de sítio para Pinus elliottii e Pinus taeda desenvolvidas no Sul do Brasil. Ciência Florestal, v.12, n.1, p.143-152, 2002. Disponível em: <http://www.ufsm.br/cienciaflorestal/artigos/ v12n1/A14V12N1.pdf>. Acesso em: 5 jun. 2011.

TONINI, H. et al. Crescimento da teca (Tectona grandis) em reflorestamento na Amazônia Setentrional. Pesquisa Florestal Brasileira, n.59, p.05-14, 2009. Disponível em: $<$ http://www.cnpf.embrapa.br/pfb/index.php/pfb/article/ download/11/39>. Acesso em: 5 jun. 2011 . doi: 10.4336/ 2009.pfb.59.05.

VARGAS-LARRETA, B. et al. Construcción de curvas dinámicas de índice de sitio para Pinus cooperi blanco. Revista Fitotecnia Mexicana, v.33, n.4, p.343-351, 2010. Disponível em: <http:/ /redalyc.uaemex.mx/redalyc/pdf/610/61015520008.pdf $>$. Acesso em: 5 jun. 2011. doi: 10.1590/S010006832006000500001 . 